\title{
Comunidades virtuais de aprendizagem em espaços de educação a distância ${ }^{1}$
}

Patrícia B. Scherer Bassani, Universidade Feevale, patriciab@feevale.br Jorge Aranda, Universidade Feevale, jorgearanda@feevale.br Daniel Alvarez, BIC FAPERGS/Feevale, danielambr@gmail.com

Resumo: Este trabalho se insere nos estudos que vêm sendo realizados na área de educação à distância (EAD), enfocando as comunidades virtuais de aprendizagem (CVA). Conforme estudos atuais, a CVA constitui o espaço onde se dá a aprendizagem online. Para fins deste estudo, entende-se que uma comunidade virtual se estabelece a partir de uma rede social mediada pelo computador. Neste trabalho, busca-se compreender a dinâmica de uma comunidade virtual de aprendizagem à luz dos estudos relacionados à área de análise de redes sociais. Para tanto, este estudo, de abordagem qualitativa, tem por objetivo mapear a rede social que se constitui em um fórum de discussão, a fim de buscar subsídios para a compreensão das CVA em espaços formais de educação à distância. Resultados parciais apontam que redes sociais distribuídas evidenciam maior equilíbrio nas interações.

Palavras-chave: educação a distância; comunidade virtual de aprendizagem; rede social.

Title: Virtual learning communities in distance education

Abstract: This work is part of the ongoing studies in the area of distance education, focusing on virtual learning communities (VLC). According to present studies, the VLC is the space where online learning happens. In our study, we assume that a virtual community is established from a social network mediated by the computer. In this paper, we try is to understand the dynamics of a VLC, based on studies related to social networks analysis. Thus, this study, based in a qualitative approach, aims to map the social network in a discussion forum, in order to understand the VLC in formal distance learning spaces. Partial results point out that distributed social networks show a higher balance in the interactions.

Keywords: distance education; virtual learning community; social network.

\section{Introdução}

De acordo com a legislação brasileira, a educação à distância (EAD) é uma "modalidade educacional na qual a mediação didático-pedagógica nos processos de ensino e aprendizagem ocorre com a utilização de meios e tecnologias de informação e comunicação, com estudantes e professores desenvolvendo atividades educativas em lugares ou tempos diversos" (DECRETO No 5.622).

Conforme o documento intitulado "Referenciais de qualidade para a educação superior à distância” (MEC, 2009), material elaborado pelo Ministério da Educação/Secretaria de Educação a Distância, a partir de uma discussão pública com especialistas na área e universidades, o uso da tecnologia aplicada à educação deve estar embasada em uma filosofia de aprendizagem que proporcione efetiva interação no processo de ensino-aprendizagem. E ainda, “como estratégia, a interação deve

${ }^{1}$ Este trabalho foi apresentado no XII Seminário Internacional de Educação - Educação e Tecnologia: significados e tendências na construção do conhecimento, na Universidade Feevale, Novo Hamburgo, RS. 
proporcionar a cooperação entre os estudantes, propiciando a formação de grupos de estudos e comunidades de aprendizagem".

Conforme estudos atuais na área de educação à distância, a comunidade virtual de aprendizagem (CVA) constitui o espaço onde se dá a aprendizagem online (Palloff e Pratt, 2002, Daniel et al., 2003). Assim, neste estudo entende-se que a compreensão do processo de constituição e permanência de uma CVA perpassa pela análise da interação entre os participantes.

A atividade de aprendizagem em uma comunidade caracteriza-se essencialmente pela conversação entre os sujeitos participantes. A conversação não se restringe às palavras, mas envolve também imagens, vídeo, som e outros. Entretanto, neste estudo se levou em conta as interações que se constituem no fórum de discussão, a partir da linguagem escrita (Downes, 2009).

Para tanto, este estudo, de abordagem qualitativa, tem por objetivo mapear a rede social que se constitui em um fórum de discussão, a fim de buscar subsídios para a compreensão das CVA em espaços formais de educação à distância. Parte-se de uma discussão sobre os conceitos de comunidades virtuais, comunidades virtuais de aprendizagem e redes sociais, para, posteriormente, apresentar a trajetória da pesquisa.

\section{Comunidades virtuais e redes sociais}

O termo comunidade remete a agrupamentos que se sentem unidos por algum tipo de sentimento de pertença, seja no sentido geográfico, por proximidade territorial ou pela busca de interesses comuns, em uma perspectiva extraterritorial. $\mathrm{O}$ desenvolvimento tecnológico, especialmente a disseminação do acesso à Internet, permitiu novas formas de comunicação entre as pessoas, sejam de localidades próximas ou distantes, oportunizando a criação de uma nova comunidade, sem base territorial. Assim, ao final do século XX e início do século XXI percebe-se a consolidação do conceito de comunidades virtuais (BAUMAN, 2003, CASTELLS, 2003).

Para Rheingold (1996), "as comunidades virtuais são os agregados sociais surgidos na Rede, quando os intervenientes de um debate o levam por diante em número e sentimento suficientes para formarem teias de relações sociais no ciberespaço" (p. 18). Recuero (2008, p. 65) ressalta que "o conceito de comunidade virtual é uma tentativa de explicar os agrupamentos sociais surgidos no ciberespaço. Trata-se de uma forma de tentar entender a mudança da sociabilidade, caracterizada pela existência de um grupo social que interage através da comunicação mediada pelo computador".

Castells (2003) apresenta a noção de comunidades virtuais, como novos suportes tecnológicos para a sociabilidade, diferentes de outras formas de interação, mas não inferiores. Destaca, também, que as comunidades virtuais trabalham com base em duas características fundamentais comuns:

a) valor da comunicação livre, horizontal, caracterizada pela comunicação online de muitos para muitos;

b) formação autônoma de redes, que envolve a possibilidade de qualquer pessoa escolher/definir os fluxos de navegação na rede, além do potencial de criar e divulgar suas próprias redes.

As comunidades virtuais com foco na aprendizagem podem ser classificadas em comunidades virtuais de aprendizagem (CVA) ou comunidades de prática (CP) (Palloff e Pratt, 2002, Daniel et al, 2003). A principal diferença entre uma CVA e uma CP consiste na natureza da participação dos sujeitos. Enquanto a CVA enfoca objetivos educacionais, a CP enfatiza o compartilhamento de experiências e interesses relacionados a atividades profissionais. Embora toda comunidade virtual tenha relação 
com um elemento de aprendizagem, nem toda comunidade pode ser chamada de CVA, pois esta implica que seus membros tenham objetivos explícitos vinculados à aprendizagem (Daniel et al., 2003).

Palloff e Pratt (2002) sustentam que a CVA constitui o espaço onde se dá a aprendizagem online. As autoras apontam alguns indicadores de que uma comunidade online está em formação:

a) interação ativa, em relação ao conteúdo do curso e à comunicação interpessoal, ou seja, para que um aluno seja considerado "presente" em aula, não basta acessar a aula, é preciso participar enviando suas reflexões/idéias;

b) aprendizagem colaborativa, percebida por meio das trocas de mensagens e/ou comentários entre os alunos e entre estes e o professor;

c) significado construído socialmente, evidenciado a partir de discussões envolvendo acordos ou questionamentos, à medida que a aula/curso vai se desenvolvendo;

d) compartilhamento de recursos (livros, artigos encontrados na Internet, fontes de pesquisa) entre alunos, possibilitando ampliar a bibliografia para além dos textos selecionados pelo professor;

e) troca de expressões de estímulo entre alunos e vontade de avaliar criticamente os trabalhos dos colegas.

Castells (2003) destaca o "deslocamento da comunidade para a rede como forma central de organizar a interação" (p. 106). Assim, as redes sociais vêm incorporando as comunidades de aprendizagem.

A partir dos estudos de Recuero (2009) uma rede social é composta por dois elementos: atores (pessoas, instituições ou grupos; os nós da rede) e suas conexões (interações ou laços sociais). A autora afirma que as "redes são metáforas estruturais" (p. 56), onde o intercâmbio e o grau de cooperação podem ser visualizados por meio de grafos representativos dos nodos de conexão.

Barabási (2003) apresenta o estudo feito por Paul Baran (1964), que identifica três topologias básicas para redes sociais: centralizada, descentralizada e distribuída (figura1).
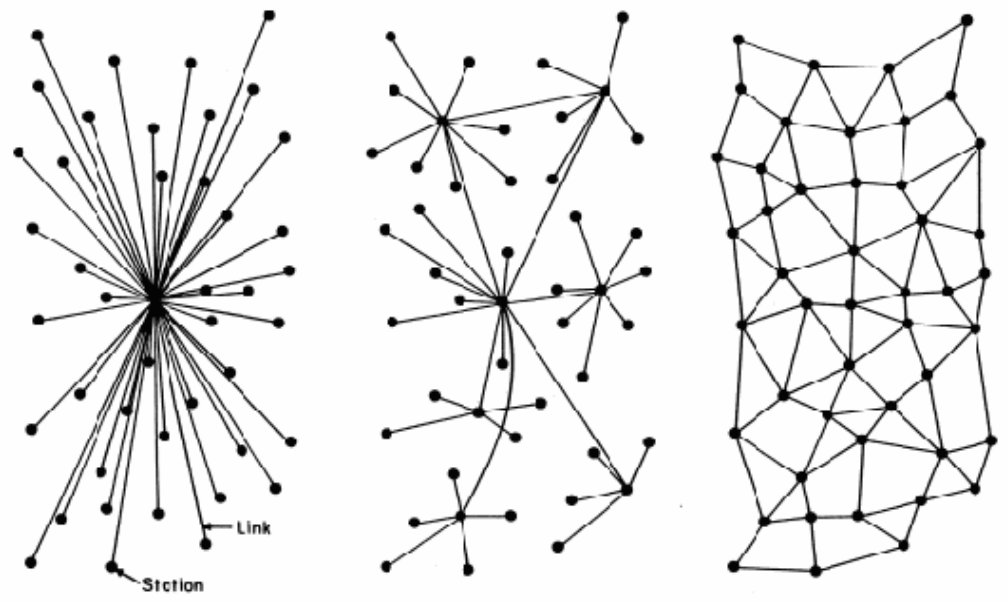

Figura 1 - Topologias das redes de Paul Baran

Fonte: Barabási (2003, p. 145)

Em uma topologia centralizada, um nó centraliza a maior parte das conexões; um padrão descentralizado possui vários centros, onde um grupo pequeno de nós conecta vários outros grupos; em um modelo distribuído, todos os nós possuem mais ou menos a mesma quantidade de conexões. 
Assim, uma rede pode ser definida como uma forma simbólica para observar os padrões das conexões estabelecidas entre os diversos atores. Então, o estudo das redes sociais é caracterizado pela busca da origem das estruturas sociais, identificando seus tipos, seus elementos, processos dinâmicos e com base em suas interações são gerados fluxos de informações que servem como material para estudos das mesmas.

As relações sociais atuam na construção dos laços sociais (RECUERO, 2009). Os laços sociais são entendidos como a efetiva interação entre os participantes de uma relação. Os laços podem ser fortes ou fracos. Laços fracos caracterizam-se pela proximidade, intimidade e intencionalidade em manter a conexão com o outro. Por outro lado, os laços fracos caracterizam-se por relações esparsas, que não traduzem intimidade e proximidade.

Recuero (2009) propõe que as comunidades virtuais na Internet possam ser reconhecidas a partir de três diferentes tipos: comunidades de associação, comunidades emergentes ou comunidades híbridas. Uma comunidade emergente caracteriza-se por um núcleo mais denso, onde estão os atores conectados por nós mais fortes, e uma periferia, onde estão os nós mais fracos. Assim, "os laços que conectam os atores na comunidade emergente são fortes no centro e fracos na periferia” (p. 154). Por outro lado, as comunidades de associação (ou de filiação) caracterizam-se essencialmente pela "associação de atores através de interação social reativa (associar-se ao grupo e ser aceito pelo mesmo), que não pressupõe interação direta entre os atores, ou mesmo interação social no sentido de conversação" (p. 156). As comunidades híbridas possuem características dos dois tipos (emergentes e associativas).

Entende-se que o fórum de discussão representa uma rede do tipo emergente, uma vez que se efetiva por meio das interações entre os diferentes atores. Nestas redes as conexões entre os nós se constituem a partir das trocas sociais realizadas pela interação e conversação, possibilitada pela comunicação mediada pelo computador.

\section{Delineando a pesquisa}

Este estudo, de natureza qualitativa, constitui uma etapa de pesquisa que busca analisar os processos que envolvem a constituição de CVA em espaços de educação à distância, especialmente no fórum de discussão.

Estudos anteriores (Bassani 2009, 2010) envolvendo a análise das interações em um fórum de discussão tiveram como objetivo investigar características da conversação em um fórum de discussão que impulsionam os processos que envolvem a constituição e permanência de CVA em espaços formais de educação à distância. A metodologia de pesquisa envolveu três momentos: análise do conteúdo das mensagens; mapeamento da interação, a partir da identificação da estrutura do fórum; e análise da relação entre o conteúdo da mensagem e a estrutura do fórum.

O conteúdo das mensagens foi analisado à luz do referencial teórico proposto por Dolle (1993) e revisitado por Behar et al (2005). Behar et al aponta quatro eixos conceituais para a análise das interações em um ambiente virtual de aprendizagem:

a) epistemológico: envolve tudo o que faz referência e/ou caracteriza o processo de construção do pensamento sobre o objeto de estudo, neste caso, o conteúdo/matéria do curso;

b) tecnológico: envolve tudo o que faz referência ao gerenciamento dos aspectos tecnológicos, como funcionamento, regras e lógica do sistema computacional e demais softwares de apoio, e o conhecimento necessário para a comunicação, interação e pertinência nestes ambientes (aspectos operacionais e funcionais);

c) social: tudo o que envolve o processo de construção numa coletividade, seja essa através de relações individuais ou interindividuais; 

sentimentos.

d) afetivo: caracteriza-se pela expressão de emoções, como desejos, emoções e

A análise das interações em um fórum de discussão permitiu evidenciar a existência de três diferentes situações em relação à articulação da conversação no fórum (Bassani, 2009):

a) Sem interação: quando os diferentes sujeitos envolvidos na discussão postam suas mensagens de forma isolada (figura 2).

\section{Enunciado 1}

Enunciado 2

Enunciado 3

\section{Figura 2 - Sem interação}

b) Interação sem articulação: quando a discussão se encontra vinculada a um mesmo enunciado, mas não existe articulação entre as diferentes mensagens; os envolvidos participam do mesmo tópico da discussão, mas cada um posta sua mensagem, caracterizando diferentes respostas para um questionamento inicial (figura $3)$.

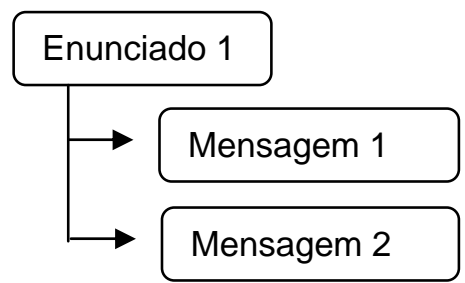

Figura 3 - Interação sem articulação

c) Interação com articulação: esta situação ficou evidenciada quando a discussão se encontra vinculada a um mesmo enunciado e as mensagens estão articuladas, conforme representado na figura 4 abaixo.

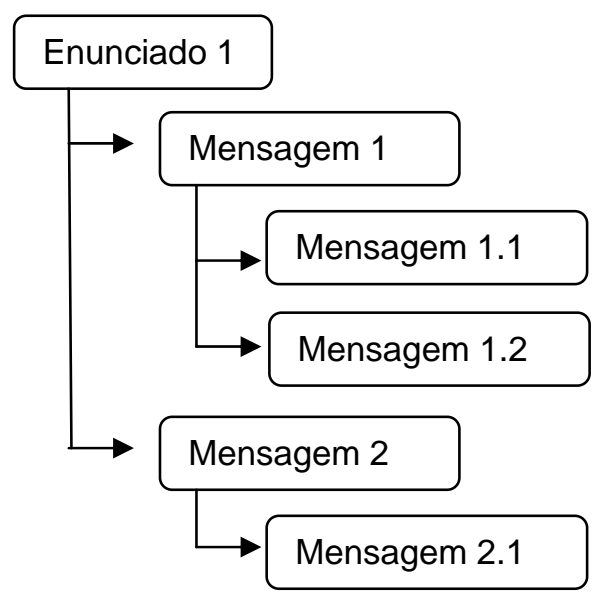

Figura 4 - Interação com articulação 
A partir do levantamento da estrutura da discussão no fórum e dos eixos conceituais das mensagens, buscou-se analisar a (possível) relação entre o tipo de mensagem postada e a continuidade das trocas. Foi possível perceber que a situação aqui denominada "sem interação" e "interação sem articulação" tende a ocorrer quando as mensagens são de cunho epistemológico. Por outro lado, foi possível perceber que a situação chamada "interação com articulação" se evidencia especialmente pela presença de unidades de registro do tipo epistemológico-social ou social-afetivo.

Assim, partindo-se desses resultados preliminares, uma segunda etapa da pesquisa busca analisar a relação entre a estrutura do fórum de discussão e o mapeamento da rede social. O mapeamento da rede social foi realizado a partir do software AGNA, que permite a visualização da rede por meio de um gráfico. A imagem é gerada automaticamente a partir dos dados inseridos em uma tabela, representando as interações entre os sujeitos.

\subsection{Mapeamento da rede social}

Conforme dito anteriormente, estudos anteriores evidenciaram três diferentes situações em relação à articulação da conversação no fórum: sem interação, interação sem articulação e interação com articulação (Bassani 2009, 2010).

O mapeamento da rede social do fórum caracterizado como "sem interação", mostra o isolamento das falas na conversação. Neste caso, este modelo de fórum, essencialmente baseado em textos de cunho epistemológico, não possibilitou a efetivação de uma rede, conforme demonstra a figura 5 .

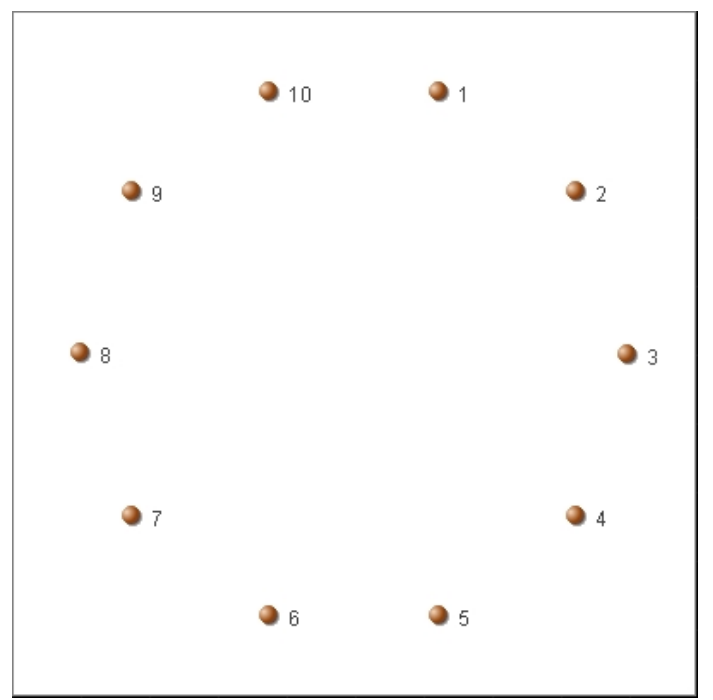

Figura 5 - Representação baseada no modelo "sem interação"

Por outro lado, percebe-se que a rede social que se constitui a partir de um fórum caracterizado pela "interação sem articulação" tende a gerar uma rede social centralizada, onde um nó centraliza quase todas as conexões. No exemplo apresentado na figura 6, o professor centraliza a discussão. 


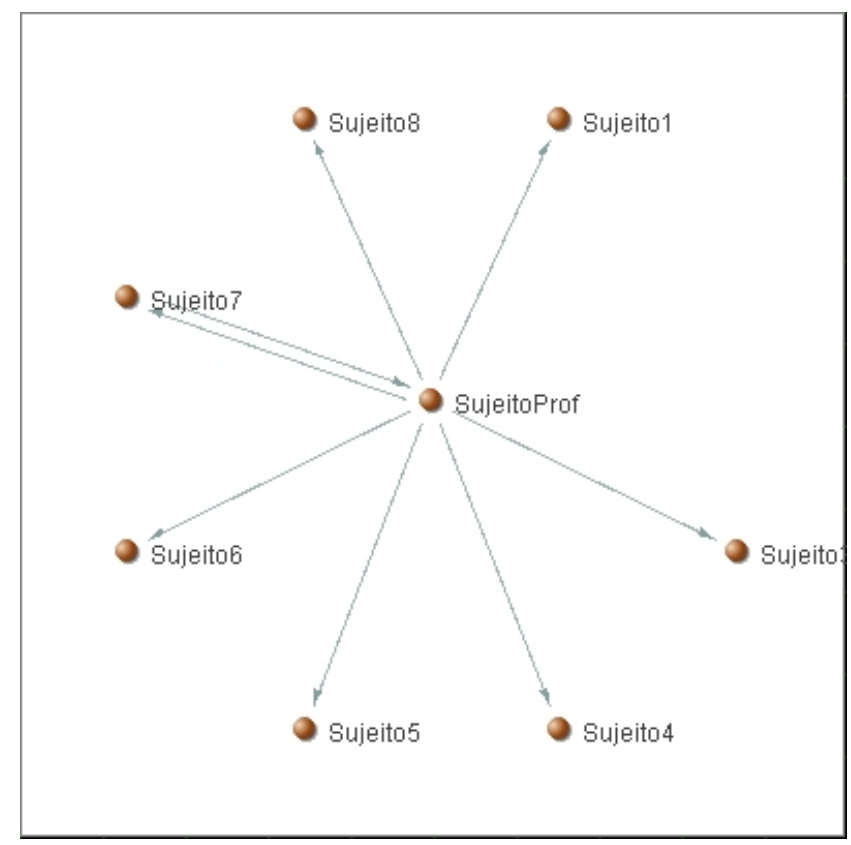

Figura 6 - Representação baseada no modelo "interação sem articulação"

Importante destacar que os estudos apontam que quanto mais conexões um nó possui, maiores as chances de ele ter mais novas conexões. Barabási chamou essa característica de preferential attachment ou conexão preferencial: "um novo nó tende a se conectar com um nó pré-existente, mas mais conectado". Isto implica na compreensão de que as redes não são constituídas de nós igualitários, ou seja, estas redes tendem a possuir nós altamente conectados e uma grande maioria de nós com poucas conexões (RECUERO, 2009).

Um aspecto essencial para a consolidação de comunidades virtuais (ou redes sociais) é o sentimento de confiança mútua entre os sujeitos participantes, que está diretamente relacionada com a capacidade que cada sujeito teria de entrar em relação com os outros, percebê-los e incluí-los em seu universo de referência (COSTA, 2005).

Assim, no modelo apresentado na figura 6, percebe-se uma centralização nas interações professor-aluno e poucas iniciativas de interação aluno-aluno, evidenciando a excessiva importância do professor na discussão.

No fórum caracterizado como "interação com articulação" percebe-se a constituição de uma rede social distribuída, onde os nós possuem mais ou menos as mesmas conexões, conforme mostra a figura 7. Neste caso, pode-se evidenciar maior equilíbrio entre as interações entre aluno-professor e aluno-aluno, caracterizando o que Palloff e Pratt (2002) entendem por interação ativa. Este modelo também vai ao encontro das características apontadas por Castells (2003) para uma comunidade virtual, evidenciando a comunicação horizontal e a formação autônoma de redes. 


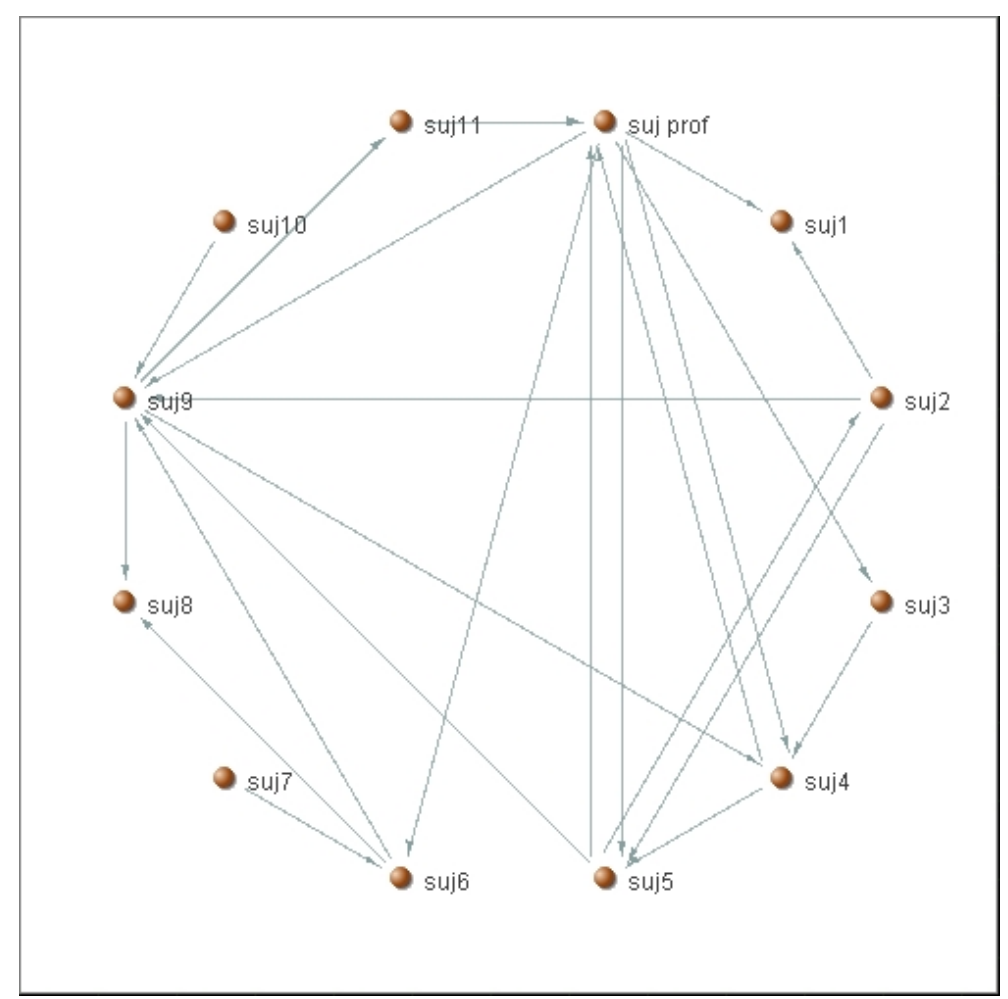

Figura 7 - Representação baseada no modelo "interação com articulação"

Recuero (2009) aponta que estas topologias são interessantes para o estudo das redes, mas apresentam limitações, uma vez que se tratam de modelos fixos e que uma mesma rede social pode ter características de vários deles.

\section{Considerações finais}

O mapeamento da rede social no fórum de discussão constitui importante ponto referencial para a compreensão dos processos que envolvem a constituição de CVA, uma vez que possibilita a visualização das relações entre os diferentes sujeitos. A partir dos estudos realizados até o momento foi possível verificar que as redes sociais distribuídas evidenciam maior equilíbrio nas interações entre professor-aluno e alunoaluno, o que vai ao encontro dos estudos na área de comunidades virtuais de aprendizagem.

Recuero (2009, p. 37) afirma que “a idéia de relação social é independente de seu conteúdo". Para a autora, o conteúdo "constitui-se naquilo que é trocado através das trocas de mensagens e auxilia a definir a relação" (p. 37).

Entende-se que o conteúdo das mensagens fornece subsídios importantes para analisar o tipo de mensagem que impulsiona as trocas interindividuais. Estudos anteriores (Bassani, 2009) apontam que a ocorrência de mensagens vinculadas aos eixos social e afetivo pode estar intimamente relacionada à constituição e permanência de uma CVA. Entretanto, apenas a identificação do tipo da mensagem apresenta poucos indicadores para um estudo que busca perceber como se constituem as CVA (Bassani, 2009).

Por outro lado, o mapeamento da rede social permite visualizar a intensidade das trocas e a força dos laços sociais, mas entende-se que este modelo também apresenta fragilidades quando analisado isoladamente, pois não permite explicitar o que diferencia a quantidade/intensidade das conexões entre os nós das redes. 
Assim, trabalhos futuros envolvem estudos sobre a relação entre o conteúdo das mensagens do fórum e a intensidade das conexões e dos laços sociais. Entende-se que os resultados desta pesquisa podem contribuir com pontos referenciais para 0 desenvolvimento de cursos à distância, especialmente no âmbito de metodologias para aprendizagem colaborativa no fórum de discussão.

\section{Referências}

BARABÁSI, Albert László. Linked: how everything is connected to everythig else and what it means for business, science, and everyday life. New York, USA: Plume, 2003.

BARDIN, L. Análise de Conteúdo. Portugal: Edições 70, 2007.

BASSANI, Patrícia. B. Scherer. Trocas interindividuais no fórum de discussão: um estudo sobre as comunidades de aprendizagem em espaços de educação à distância In: XX Simpósio Brasileiro de Informática na Educação (SBIE 2009), 2009, Florianópolis. BASSANI, Patrícia. B. Scherer. Reflexões sobre a constituição de comunidades virtuais de aprendizagem em espaços de educação à distância. In: XI Conferência Internacional de Educação em Engenharia e Tecnologia - INTERTECH'2010, 2010a, Ilhéus - BA.

BASSANI, Patrícia. B. Scherer et al. A força dos laços sociais na constituição de uma comunidade virtual de aprendizagem. In: XI Conferência Internacional de Educação em Engenharia e Tecnologia - INTERTECH'2010, 2010b, Ilhéus - BA.

BAUMAN, Zygmunt. Comunidade: a busca por segurança no mundo atual. Rio de Janeiro, RJ: Jorge Zahar, 2003.

BEHAR, P, et al. A definição de Eixos Conceituais e Indicadores para uma metodologia didático-pedagógica voltada para ambientes virtuais de aprendizagem. In: X Seminário de educação, tecnologia e sociedade, 2005, Taquara.

BRASIL. DECRETO No 5.622, DE 19 DE DEZEMBRO DE 2005. Disponível em: < http://www.planalto.gov.br/ccivil_03/_Ato2004-2006/2005/Decreto/D5622.htm>.

Acesso em: 26 de abr. 2010.

CASTELLS, M.. A Galáxia da Internet. Rio de Janeiro: Jorge Zahar Ed., 2003.

COSTA, R. Por um novo conceito de comunidade: redes sociais, comunidades pessoais, inteligência coletiva. Interface (Botucatu) [online]. 2005, vol.9, n.17, pp. 235-248.

DANIEL, B., SCHWIER, R., MCCALLA, G. Social capital in virtual learning communities and distributed communities of practice. Canadian Journal of Learning and Technology. V. 29(3), Fall, 2003.

DOLLE, J, M. Para além de Freud e Piaget: referenciais para novas perspectivas em psicologia. Petrópolis, RJ: Vozes, 1993.

DOWNES, S. Learning networks in practice. Disponível em: http://partners.becta.org.uk/upload-

dir/downloads/page_documents/research/emerging_technologies07_chapter2.pdf.

Acesso em jun. 2009.

MEC. Referenciais de qualidade para a educação superior à distância. Disponível em: http://portal.mec.gov.br/seed/arquivos/pdf/legislacao/refead1.pdf. Acesso em jun, 2009.

PALLOFF, R, PRATT, K. Construindo comunidades aprendizagem no ciberespaço. Porto Alegre: Artmed, 2002.

RECUERO, R. Comunidades em redes sociais na Internet: Um estudo de caso dos fotologs brasileiros. Liinc em Revista, v.4, n.1, março 2008. Rio de Janeiro. p.63-83.

RECUERO, R. Redes sociais na Internet. Porto Alegre: Sulina, 2009.

RHEINGOLD, H. A comunidade virtual. Lisboa: Gradiva Publicações, 1996. 